\title{
Growth Parameter Optimization and Biochemical Appraisals of Spirulina platensis
}

\author{
V.P. Joshi ${ }^{1 *}$, P.M. Chauhan ${ }^{1}$, K.B. Joshi ${ }^{2}$ and N.H. Joshi ${ }^{3}$ \\ ${ }^{1}$ Department of Renewable Energy Engineering, CAET, ${ }^{2}$ Department of Biochemistry, COA, \\ ${ }^{3}$ Department of Aquaculture, COF, Junagadh Agricultural University, \\ Junagadh-362001 (Gujarat), India \\ *Corresponding author
}

A B S T R A C T

Keywords

Algal species, Growth Parameter optimization, Photobioreactor, Protein, Secondary metabolites, Lipid

Article Info

Accepted: 04 October 2018 Available Online: 10 November 2018
Various microalgae species have high amount of protein and lipid level that is why this is now a days consider as one of the main reasons of an unconventional source of this compound. Spirulina platensis is one of the richest protein sources of microbial origin, having similar protein levels when compared to pulse and animal protein sources. The use of Spirulina platensis in food can bring benefits to human health owing to its chemical composition, as it has high levels of vitamins, minerals, secondary metabolites, essential fatty acids, amino acids and pigments. Furthermore, the development of new protein sources to supply the shortage of this nutrient is an urgent need and protein from Spirulina platensis plays an important role in such scenario. This investigation was carried out for growth parameter optimization of Spirulina platensis cultivating in laboratory scale photobioreactor. Combination of three different growth medium, temperature and light intensity respectively studied. The optimum growth condition of Spirulina platensis was found for BG-11 growth medium with temperature $30^{\circ} \mathrm{C}$ and light intensity factor $50 \%$ (in this case 10000 lux). Biochemical analysis was also carried out in this study and it was conclude that Spirulina platensis was found rich in protein content.

\section{Introduction}

Microalgae are microscopic photosynthetic organisms that are found in both marine and fresh water environments. Their photosynthetic mechanism is similar to landbased plants, due to a simple cellular structure, and the fact that they are submerged in an aqueous environment, where they have efficient access to water, $\mathrm{CO}_{2}$ and other nutrients; they are generally more efficient in converting solar energy into biomass. There is a significant variation in the overall biomass productivity and resulting secondary metabolites, land use and biodiesel productivity, however, with a clear advantage for microalgae. Algae offer a diverse spectrum of valuable products and pollution solutions, such as food, nutritional compounds, omega-3 fatty acids, animal feed, energy sources 
(including jet fuel, aviation gas, biodiesel, gasoline, and bioethanol), organic fertilizers, biodegradable plastics, recombinant proteins, pigments, medicines, pharmaceuticals, and vaccines (Pulz, 2001; Pienkos and Darzins, 2009).

Microalgae have a chemical composition containing lipids, carbohydrates and proteins on a dry matter basis (DMB), which can vary depending on the genus and species, and on the growing conditions for a particular species. Several factors, including nutrient availability, temperature, $\mathrm{pH}$ and light intensity can effect microalgal chemical composition. The suitability of microalgae to produce biodiesel is due to their high lipid content. Algal species have higher growth rates under natural conditions and higher biomass productivities compared to oil-seed crops in terms of land area requirement. Protein deficiency in human nutrition is a major concern for developing countries; thus there is a need to be in search of diversifies protein sources and finding of new unconventional ones. The high protein concentration of various microalgal species makes them an interesting alternative source as nutrient supplement. The demand for sustainable energy has led to increased interest in biomass energy from microalgae.

In recent years, microalgae have been considered a versatile raw material for production of biofuels, such as biodiesel, bioethanol, biogas and bio-hydrogen, owing to their favorable composition. Algal growth can high reach densities to achieve greater levels of light harvest within a few days and are hence considered economic and efficient. Therefore, scientist began to pay more attention to develop microalgae for extracting more sustainable energy. A strategy it is to be done to have higher biomass yield than high protein and lipid content of algal cells (achieved usually by inducing stress conditions). Higher biomass yield can pave ways for other possible routes of useful secondary metabolites and energy generation using many other biofuels by employing various other bio-chemical processes and also by product utilization.

\section{Materials and Method}

\section{Microalgae strain and pre-cultivation conditions}

Based on literature reviewed and local species availability fresh water strain i.e Cyanobacteria phylum Spirulina Platensis had been selected for cultivation. Pure cultures of Spirulina Platensis was procured and identified morphologically by Dr. N. H. Joshi, Fisheries research center, Okha, Junagadh Agricultural University, Gujarat. This microalgae strain was cultivated in laboratory scale closed photobioreactor (3 L) for growth parameter analysis. The stock culture of all the strain was maintained regularly on agar slants using the sterilized BG-11, CHU and Walne medium (with initial $\mathrm{pH}$ of 6.9) under laboratory conditions at. Mediums were autoclaved at $121{ }^{\circ} \mathrm{C}$ for 20 minutes in autoclave. All the strains were transferred from agar slant into a liquid medium and incubated at $24{ }^{\circ} \mathrm{C} \quad\left( \pm 1{ }^{\circ} \mathrm{C}\right)$ under light intensity ( 10,000 Lux) with 16: 8 light dark cycle in Incubator. The serial transfer of all agar slants and liquid cultures into new medium was performed in a UV light sterilized laminar air flow hood to avoid the possibility of contamination attack. Liquid cultures were used as the source of inoculums for the designed experiments and cultured in photobioreactor. The 3 liter laboratory scale photobioreactor used for stock culture were shown in Figure 1. The morphology of pure strains was regularly examined under an optical microscope under $100 \times$ and photographed by a ZESIS Axio Imager 2 microscope. 
Factors affecting growth of microalgae culture

Various factors affecting growth of Spirulina Platensis strain were studied at laboratory scale photobioreactor. The following growth factors were evaluated and further analyzed in laboratory scale photobioreactor.

Temperature

Media

Light supply

\section{Growth parameter optimization}

The treatments consist of three levels of temperature, three levels of media and three levels of light intensity to evaluate effect of growth parameters on Spirulina Platensis cultivation. This experiment was conducted with 27 treatment combinations given in Table 1. Here in laboratory scale photobioreactor the light unit was provided with capacity was 20,000 lux. The light unit was provided with pulse modulation control to control its light intensity. For this study light intensity were considered as $100 \%$ means 20,000 lux whereas $50 \%$ means 10,000 lux and 6000 lux means $30 \%$ light intensity respectively.

\section{Cultural media composition}

Three cultural medium i.e. BG-11, Chu and Walne were prepared based on computability for particular species for better growth cultivation as per standard composition.

\section{Microscopic and biochemical characterization of microalgae species}

\section{Microscopic characterization}

\section{Cell counting trough Haemocytometer (Guillard and Sieracki, 2005)}

Cells were counted with haemocytometer observing in microscope. Counting cells in cultures by any means has fundamental principle to estimate the size of the cultured population, expressed as the total number of cells (colonies, occasionally) in the culture as a whole or, more usually, as individuals per unit volume of culture. Microalgal strains cell counting was analyzed with standard procedure for cell count by haemocutometer as indicated in Figure 2.

The growth measurement of microalgae was carried out with UV-visible spectrophotometer (Evolution 260 Bio). The efficiency of biomass growth was controlled by measuring the optical density (OD), which is defined as the absorption of visible radiation. (Absorption peak of chlorophyll is at about $680 \mathrm{~nm})$. The optimal wavelength $(686 \mathrm{~nm})$ was determined based on the UV spectrum. Growth rates of Spirulina platensis was calculated with the doubling time or the specific growth rate. The specific growth rates $\left(\mu ;\right.$ time $\left.^{-1}\right)$ were determined by measuring the doubling time in the exponential growth phase from semi-log plots of cell density or its biomass.

\section{Biochemical characterization}

Biochemical characterizations were carried out from microalgae sample obtained from centrifugal harvesting. The supernatant was discarded and remaining concentrated algae sample were collected for its further analysis. Concentrated algal samples were frozen overnight at $-20^{\circ} \mathrm{C}$. Following biochemical parameters were studied,

\section{Moisture content (\%)}

Moisture content of microalgae culture was determined as a percent on wet basis by oven drying method. The moisture content as percentage by mass of the sample was calculated from wet microalgae culture after harvesting by centrifugation method was oven dried $60{ }^{\circ} \mathrm{C}$ until one get constant weight (AOAC, 1984) 


\section{Carbohydrate estimation (Mercz, 1994)}

Carbohydrate estimation was carried out by phenol sulphuric acid method to determine the total carbohydrate content in the algal species.

\section{Protein content}

The protein content of each microalgae species was measured to determine protein content using Kjeldahl method.

\section{Chlorophyll content (Jeffrey and Humphrey, 1975)}

Chlorophyll extraction was carried out with Dimethyl formaldehyde method. This method was an easy and quick extraction method. The method results in very stable measurements.

\section{Lipid extraction (Joshi et al., 2018)}

A Soxtherm-based lipid extraction procedure was followed and optimized for the isolation of lipids from dried Spirulina platensis biomass.

\section{Results and Discussion}

Effect of growth media on cultivation of Spirulina platensis

Effect of different media on growth of Spirulina platensis in the present investigation was studied with three different media i.e BG11, CHU and Walne. The effect of different growth parameter on cell count is given in Table 1 and specific growth rate, division per day and generation time is givenin Table 2. As Spirulina platensis is a fresh water microalgae the result indicated in Table 2 that specific growth rate $\mathrm{k}$ ' was found higher in medium i.e. BG-11 medium $0.527 \pm 0.012$ followed by CHU medium 0.294 \pm 0.001 and Walne medium $0.060 \pm 0.006$. Division per day was found highest in BG-11 medium i.e.
$0.760 \pm 0.017$ followed by CHU medium $0.425 \pm 0.002$ and Walne medium $0.086 \pm 0.009$. Whereas generation time was found lowest in BG-11 medium i.e. $1.316 \pm 0.029$ followed by CHU medium 2.355 \pm 0.009 and Walne medium $11.763 \pm 1.183$.

Effect of temperature on cultivation of Spirulina platensis

Temperature plays a vital role in microalgae growth. Effect of temperature on growth of Spirulina platensis was studied. Higher growth rate were found at $30^{\circ} \mathrm{C}$ followed by $28^{\circ} \mathrm{C}$ and at $33^{\circ} \mathrm{C}$ as indicated in Figure 3 and also data given in Table 1 and 2. As indicated in Table 2 the specific growth rate k' was found higher at $30{ }^{\circ} \mathrm{C}$ i.e. $0.731 \pm 0.017$ followed by $28^{\circ} \mathrm{C}$ i.e. $0.517 \pm 0.020$ and $33^{\circ} \mathrm{C}$ i.e. $0.234 \pm 0.008$. Division per day was found highest at $30{ }^{\circ} \mathrm{C}$ i.e. $1.005 \pm 0.0024$ followed by $28^{\circ} \mathrm{C}$ i.e. $0.746 \pm 0.029$ and $33{ }^{\circ} \mathrm{C}$ i.e. $0.337 \pm 0.012$. Whereas generation time was found lowest in $30^{\circ} \mathrm{C}$ i.e. $0.948 \pm 0.022$ followed by $28^{\circ} \mathrm{C}$ i.e. $1.343 \pm 0.052$ and $33^{\circ} \mathrm{C}$ i.e. $2.969 \pm 0.105$. -The optimum temperature for growth of Spirulina platensis was found $30^{\circ} \mathrm{C}$.

\section{Effect of light intensity on cultivation of Spirulina platensis}

Light intensity in the present work was studied with three different levels i.e. $30 \%, 50 \%$ and $100 \%$. In this study $100 \%$ light intensity means 20,000 lux, 50\% light intensity means 10,000 lux and $30 \%$ light intensity means 6,000 lux respectively. The effect of light on growth parameters of Spirulina platensis were found highest at $50 \%$, followed by $100 \%$ and $30 \%$ light intensity as indicated in Figure 2 also computed in Table 1 and 2. As given in Table 2 specific growth rate k' was found higher at light intensity $50 \%$ i.e. $0.683 \pm 0.048$ followed by $100 \%$ i.e. $0.325 \pm 0.025$ and $30 \%$ i.e. $0.218 \pm 0.035$. Division per day was found 
highest at $50 \%$ i.e. $0.986 \pm 0.069$ followed by $100 \%$ i.e. $0.469 \pm 0.037$ and $30 \%$ i.e. $0.315 \pm 0.051$. Whereas generation time was found lowest in $50 \%$ i.e. $1.019 \pm 0.071$ followed by $100 \%$ i.e. $2.146 \pm 0.167$ and $30 \%$ i.e. $3.261 \pm 0.526$.

\section{Total carbohydrates content}

Chemically, carbohydrates were molecules that were composed of carbon, hydrogen and oxygen, made up of sugars, starches, cellulose and lignin. Carbohydrate was an essential component for metabolism and metabolic processes. (Wijffels et al., 2010) stated that algal carbohydrates can provide human health benefits in the form of anticoagulants, dietary fibers and antioxidants. The types and total carbohydrates content vary strongly according to algae species. In present study, the carbohydrate content of the Spirulina platensis was found $11.07 \%$ indicated in Table 3. As Spirulina platensis is fresh water algae and high in protein content so carbohydrate was found lower. Lipids and carbohydrate contents should obviously be very variable depending upon the state of nutrition cells (Ricketts, 1966).

\section{Protein and nitrogen content}

In the present study, quantitative analysis of protein for microalgae species Spirulina platensis was found $47.59 \mathrm{mg} . \mathrm{g}^{-1} \%$ appeared to be an interesting potential source of food proteins as it seems to have highest percentage of protein content and excellent nutrient value indicated in Table 3. Similar result was found by Spolaore et al., 2006. Spirulina platensis has now a day gained worldwide popularity as a food supplement (Colla et al., 2007).

Fig.1 Cultivation and growth paramter optimization of Spirulina platensis at laboratery scale photobioreactor

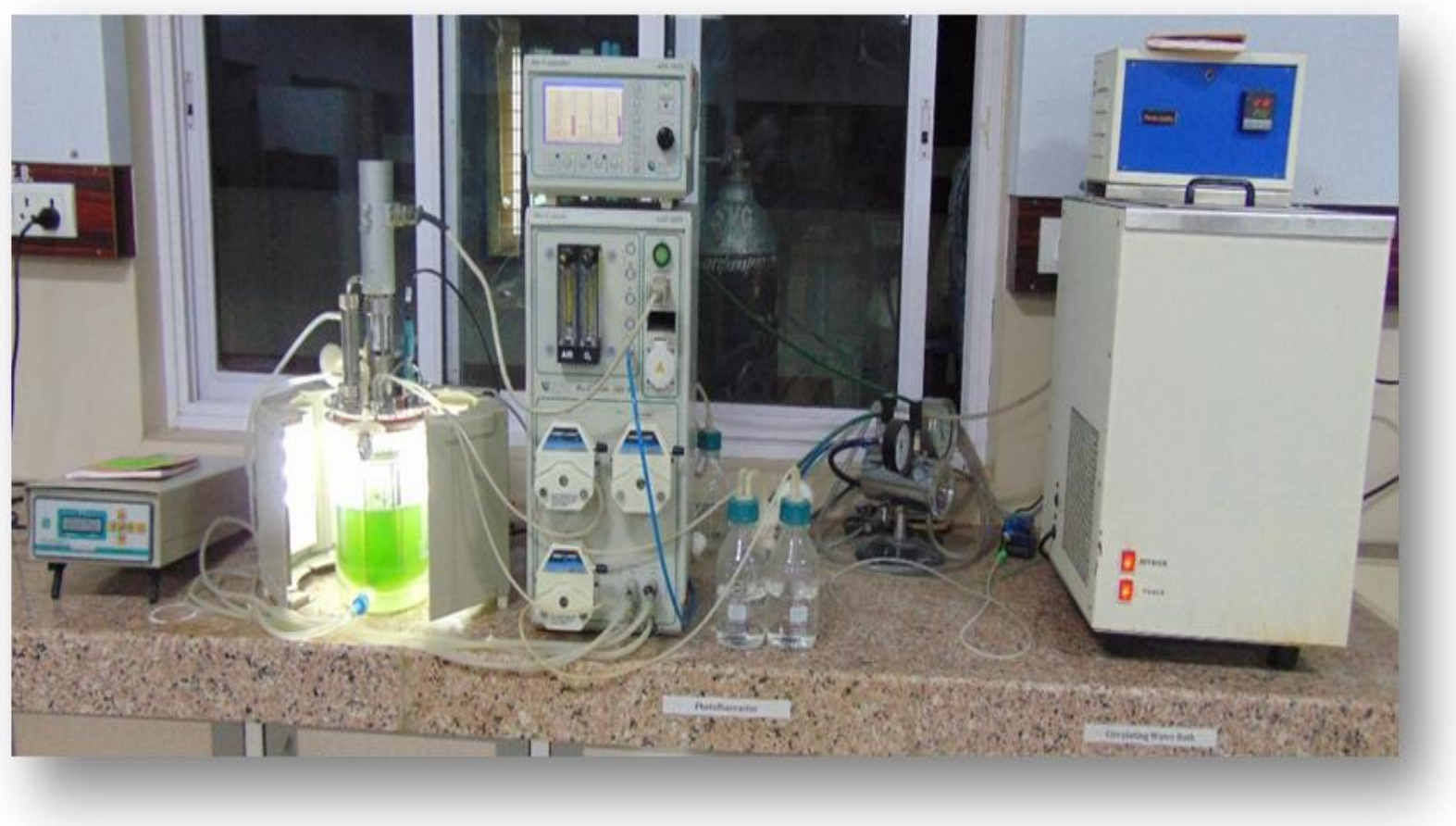


Fig.2 Microscopy view of Spirulina platensis

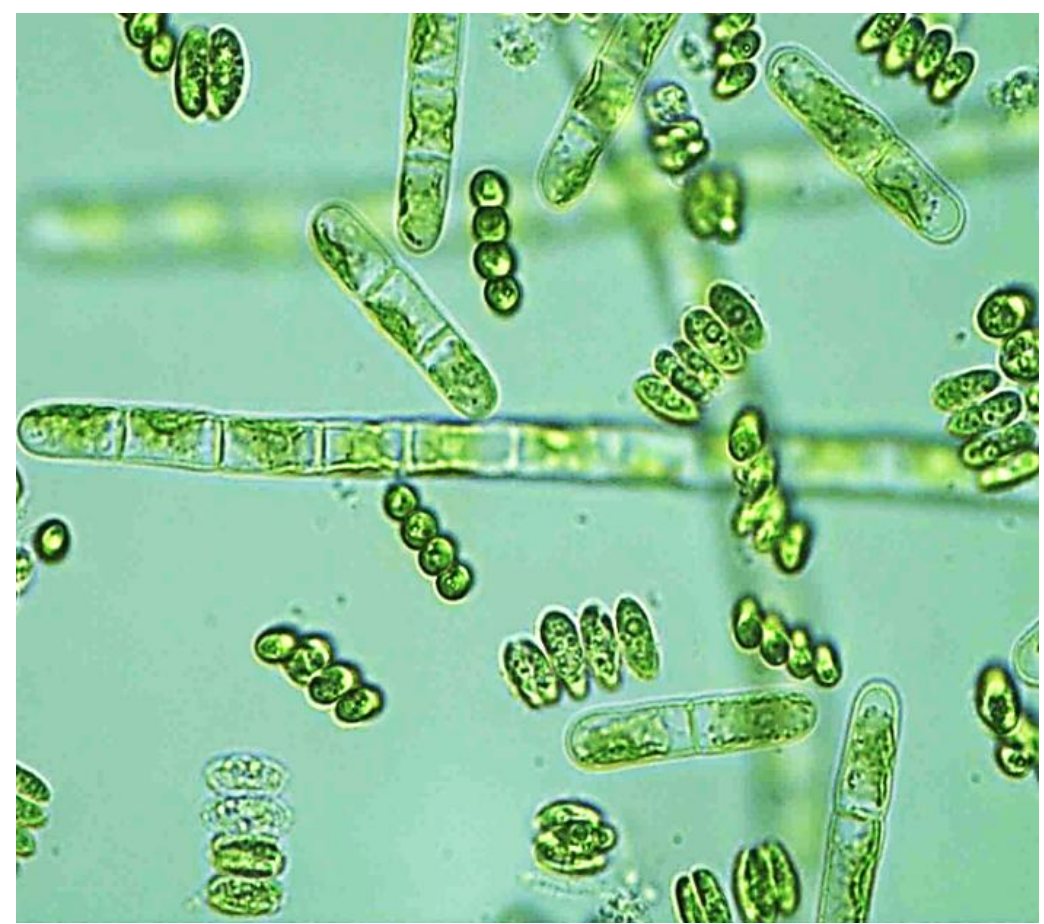

Fig.3 Effect of media, temperature and light Intensity on Spirulina platensis based on cell counting

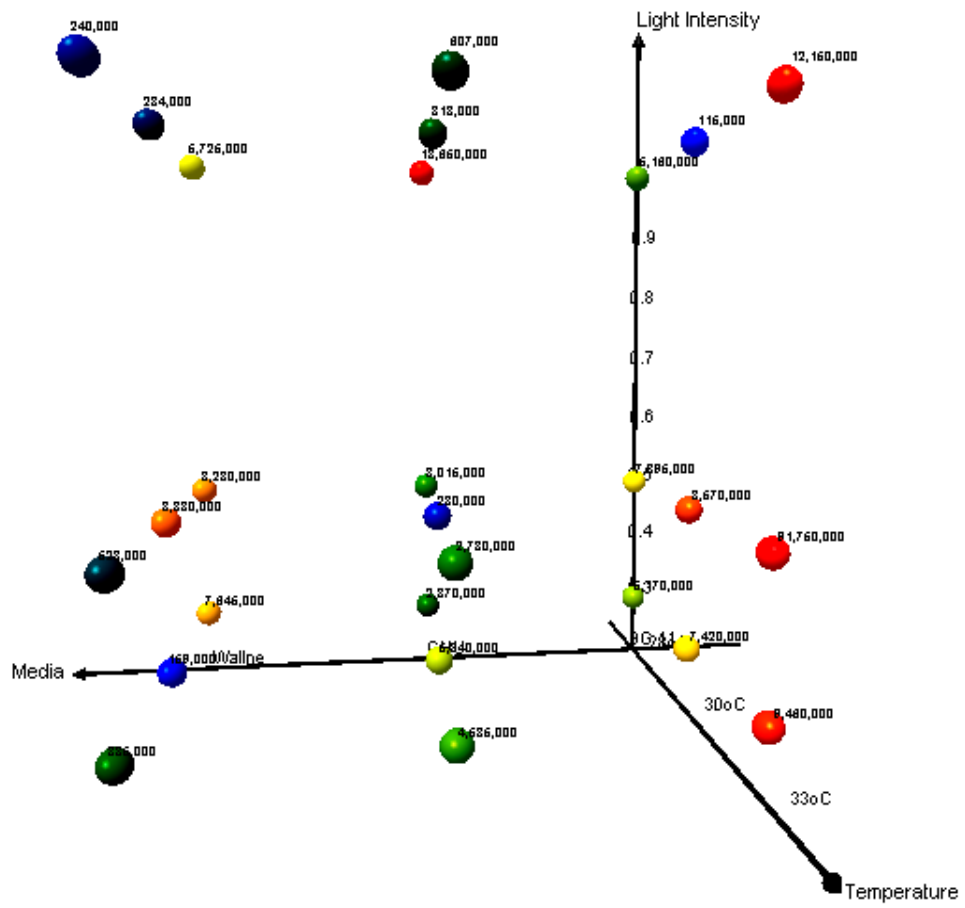

\footnotetext{
No. Of Cell $91,750,000$ $18,650,000$ $12,150,000$ $9,460,000$ $8,570,000$ $8,380,000$ $8,280,000$

$7,645,000$ $7,420,000$ $7,395,000$

$5,725,000$ $5,340,000$ $5,170,000$

$5,160,000$ $4,535,000$ $3,015,000$ $2,730,000$ $2,370,000$ 835,000 818,000 607,000 528,000 284,000 240,000 230,000

159,000 115,000

$X=$ Temperature $Y=$ Media

$Z=$ Light Intensi $\mathrm{Clr}=\mathrm{No}$. Of Cell
} 
Table.1 Effect of different growth treatment on cell count In Spirulina platensis

\begin{tabular}{|c|c|c|c|}
\hline \multicolumn{3}{|c|}{ Treatment } & \multirow{2}{*}{$\begin{array}{l}\text { Cell Count } \\
\left.(\mathbf{X 1 0})^{5}\right)\end{array}$} \\
\hline Temperature & Media & Light Intensity & \\
\hline $28^{\circ} \mathrm{C}$ & BG 11 & $30 \%$ & 51.70 \\
\hline $28^{\circ} \mathrm{C}$ & BG 11 & $50 \%$ & 73.95 \\
\hline $28^{\circ} \mathrm{C}$ & BG 11 & $100 \%$ & 51.60 \\
\hline $28^{\circ} \mathrm{C}$ & CHU & $30 \%$ & 23.70 \\
\hline $28^{\circ} \mathrm{C}$ & $\mathrm{CHU}$ & $50 \%$ & 30.15 \\
\hline $28^{\circ} \mathrm{C}$ & $\mathrm{CHU}$ & $100 \%$ & 186.50 \\
\hline $28^{\circ} \mathrm{C}$ & Wallne & $30 \%$ & 76.45 \\
\hline $28^{\circ} \mathrm{C}$ & Wallne & $50 \%$ & 82.80 \\
\hline $28^{\circ} \mathrm{C}$ & Wallne & $100 \%$ & 57.25 \\
\hline $30^{\circ} \mathrm{C}$ & BG 11 & $30 \%$ & 74.20 \\
\hline $30^{\circ} \mathrm{C}$ & BG 11 & $50 \%$ & 917.50 \\
\hline $30^{\circ} \mathrm{C}$ & BG 11 & $100 \%$ & 1.15 \\
\hline $30^{\circ} \mathrm{C}$ & $\mathrm{CHU}$ & $30 \%$ & 53.40 \\
\hline $30^{\circ} \mathrm{C}$ & $\mathrm{CHU}$ & $50 \%$ & 2.30 \\
\hline $30^{\circ} \mathrm{C}$ & $\mathrm{CHU}$ & $100 \%$ & 8.18 \\
\hline $30^{\circ} \mathrm{C}$ & Wallne & $30 \%$ & 1.59 \\
\hline $30^{\circ} \mathrm{C}$ & Wallne & $50 \%$ & 83.80 \\
\hline $30^{\circ} \mathrm{C}$ & Wallne & $100 \%$ & 2.84 \\
\hline $33^{\circ} \mathrm{C}$ & BG 11 & $30 \%$ & 94.60 \\
\hline $33^{\circ} \mathrm{C}$ & BG 11 & $50 \%$ & 85.70 \\
\hline $33^{\circ} \mathrm{C}$ & BG 11 & $100 \%$ & 121.50 \\
\hline $33^{\circ} \mathrm{C}$ & $\mathrm{CHU}$ & $30 \%$ & 45.35 \\
\hline $33^{\circ} \mathrm{C}$ & $\mathrm{CHU}$ & $50 \%$ & 27.30 \\
\hline $33^{\circ} \mathrm{C}$ & $\mathrm{CHU}$ & $100 \%$ & 6.07 \\
\hline $33^{\circ} \mathrm{C}$ & Wallne & $30 \%$ & 8.35 \\
\hline $33^{\circ} \mathrm{C}$ & Wallne & $50 \%$ & 5.28 \\
\hline $33^{\circ} \mathrm{C}$ & Wallne & $100 \%$ & 2.40 \\
\hline $28^{\circ} \mathrm{C}$ & BG 11 & $50 \%$ & 7.93 \\
\hline
\end{tabular}


Table.2 Cell density, specific growth rate, division/day and generation time (doubling time) in Spirulina platensis

\begin{tabular}{|c|c|c|c|c|c|c|c|c|c|c|c|c|}
\hline \multirow{2}{*}{$\begin{array}{l}\text { Treatment } \\
\text { s }\end{array}$} & \multicolumn{9}{|c|}{ Optical Density (O.D) } & \multirow{2}{*}{$\begin{array}{c}\text { Specific Growth } \\
\text { Rate (K') }\end{array}$} & \multirow{2}{*}{$\begin{array}{l}\text { Division } \\
\text { /Day }\end{array}$} & \multirow{2}{*}{$\begin{array}{c}\text { Generat } \\
\text { ion } \\
\text { Time }\end{array}$} \\
\hline & 0 Day & 2 Day & 4 Day & 6 Day & 8 Day & 10 Day & $\begin{array}{c}12 \\
\text { Day }\end{array}$ & $\begin{array}{c}14 \\
\text { Day }\end{array}$ & $\begin{array}{c}16 \\
\text { Day }\end{array}$ & & & \\
\hline BG-11 & $\begin{array}{c}0.032 \pm 0 \\
001\end{array}$ & $\begin{array}{c}0.064 \pm \\
0.003\end{array}$ & $\begin{array}{c}0.108 \pm \\
0.003\end{array}$ & $\begin{array}{c}0.320 \pm \\
0.001\end{array}$ & $\begin{array}{c}0.422 \pm \\
0.001\end{array}$ & $\begin{array}{c}0.567 \pm \\
0.003\end{array}$ & $\begin{array}{c}0.615 \pm \\
0.003\end{array}$ & $\begin{array}{c}0.750 \pm \\
0.005\end{array}$ & $\begin{array}{c}0.854 \pm \\
0.004\end{array}$ & $0.527 \pm 0.012$ & $\begin{array}{c}0.760 \pm \\
0.017\end{array}$ & $\begin{array}{c}1.316 \pm \\
0.029\end{array}$ \\
\hline CHU & $\begin{array}{c}0.020 \pm 0 \\
000\end{array}$ & $\begin{array}{c}0.034 \pm \\
0.001\end{array}$ & $\begin{array}{c}0.076 \pm \\
0.002\end{array}$ & $\begin{array}{c}0.100 \pm \\
0.001\end{array}$ & $\begin{array}{c}0.186 \pm \\
0.002\end{array}$ & $\begin{array}{c}0.254 \pm \\
0.002\end{array}$ & $\begin{array}{c}0.307 \pm \\
0.002\end{array}$ & $\begin{array}{c}0.385 \pm \\
0.004\end{array}$ & $\begin{array}{c}0.430 \pm \\
0.001\end{array}$ & $0.294 \pm 0.001$ & $\begin{array}{c}0.425 \pm \\
0.002\end{array}$ & $\begin{array}{c}2.355 \pm \\
0.009\end{array}$ \\
\hline Walne & $\begin{array}{c}0.025 \pm 0 \\
001\end{array}$ & $\begin{array}{c}0.043 \pm \\
0.001\end{array}$ & $\begin{array}{c}0.067 \pm \\
0.002\end{array}$ & $\begin{array}{c}0.092 \pm \\
0.002\end{array}$ & $\begin{array}{c}0.144 \pm \\
0.002\end{array}$ & $\begin{array}{c}0.188 \pm \\
0.001\end{array}$ & $\begin{array}{c}0.248 \pm \\
0.000\end{array}$ & $\begin{array}{c}0.303 \pm \\
0.002\end{array}$ & $\begin{array}{c}0.416 \pm \\
0.006\end{array}$ & $0.060 \pm 0.006$ & $\begin{array}{c}0.086 \pm 0 \\
009\end{array}$ & $\begin{array}{c}11.763 \pm \\
1.183\end{array}$ \\
\hline $28^{\circ} \mathrm{C}$ & $\begin{array}{c}0.020 \pm 0 \\
001\end{array}$ & $\begin{array}{c}0.035 \pm \\
0.002\end{array}$ & $\begin{array}{c}0.047 \pm \\
0.002\end{array}$ & $\begin{array}{c}0.069 \pm \\
0.001\end{array}$ & $\begin{array}{c}0.124 \pm \\
0.002\end{array}$ & $\begin{array}{c}0.221 \pm \\
0.002\end{array}$ & $\begin{array}{c}0.347 \pm \\
0.002\end{array}$ & $\begin{array}{c}0.454 \pm \\
0.001\end{array}$ & $\begin{array}{c}0.523 \pm \\
0.003\end{array}$ & $0.517 \pm 0.020$ & $\begin{array}{c}0.746 \pm \\
0.029\end{array}$ & $\begin{array}{c}1.343 \pm \\
0.052\end{array}$ \\
\hline $30^{\circ} \mathrm{C}$ & $\begin{array}{c}0.022 \pm 0 \\
000\end{array}$ & $\begin{array}{c}0.030 \pm \\
0.000\end{array}$ & $\begin{array}{c}0.057 \pm \\
0.002\end{array}$ & $\begin{array}{c}0.146 \pm \\
0.001\end{array}$ & $\begin{array}{c}0.268 \pm \\
0.002\end{array}$ & $\begin{array}{c}0.380 \pm \\
0.001\end{array}$ & $\begin{array}{c}0.507 \pm \\
0.004\end{array}$ & $\begin{array}{c}0.644 \pm \\
0.002\end{array}$ & $\begin{array}{c}0.715 \pm \\
0.005\end{array}$ & $0.731 \pm 0.017$ & $\begin{array}{c}1.055 \pm 0 \\
024\end{array}$ & $\begin{array}{c}0.948 \pm 0 \\
022\end{array}$ \\
\hline $33^{\circ} \mathrm{C}$ & $\begin{array}{c}0.021 \pm 0 \\
000\end{array}$ & $\begin{array}{c}0.041 \pm \\
0.001\end{array}$ & $\begin{array}{c}0.057 \pm \\
0.001\end{array}$ & $\begin{array}{c}0.086 \pm \\
0.002\end{array}$ & $\begin{array}{c}0.122 \pm \\
0.002\end{array}$ & $\begin{array}{c}0.232 \pm \\
0.001\end{array}$ & $\begin{array}{c}0.303 \pm \\
0.002\end{array}$ & $\begin{array}{c}0.387 \pm \\
0.002\end{array}$ & $\begin{array}{c}0.425 \pm \\
0.004\end{array}$ & $0.234 \pm 0.008$ & $\begin{array}{c}0.337 \pm 0 \\
012\end{array}$ & $\begin{array}{c}2.969 \pm 0 \\
105\end{array}$ \\
\hline $100 \%$ & $\begin{array}{c}0.022 \pm 0 \\
000\end{array}$ & $\begin{array}{c}0.047 \pm \\
0.002\end{array}$ & $\begin{array}{c}0.065 \pm \\
0.003\end{array}$ & $\begin{array}{c}0.097 \pm \\
0.002\end{array}$ & $\begin{array}{c}0.166 \pm \\
0.004\end{array}$ & $\begin{array}{c}0.232 \pm \\
0.001\end{array}$ & $\begin{array}{c}0.312 \pm \\
0.001\end{array}$ & $\begin{array}{c}0.387 \pm \\
0.002\end{array}$ & $\begin{array}{c}0.476 \pm \\
0.001\end{array}$ & $0.325 \pm 0.025$ & $\begin{array}{c}0.469 \pm 0 \\
037\end{array}$ & $\begin{array}{c}2.146 \pm 0 \\
167\end{array}$ \\
\hline $50 \%$ & $\begin{array}{c}0.022 \pm 0 \\
001\end{array}$ & $\begin{array}{c}0.054 \pm \\
0.002\end{array}$ & $\begin{array}{c}0.090 \pm \\
0.001\end{array}$ & $\begin{array}{c}0.133 \pm \\
0.002\end{array}$ & $\begin{array}{c}0.245 \pm \\
0.004\end{array}$ & $\begin{array}{c}0.369 \pm \\
0.001\end{array}$ & $\begin{array}{c}0.501 \pm \\
0.002\end{array}$ & $\begin{array}{c}0.585 \pm \\
0.004\end{array}$ & $\begin{array}{c}0.697 \pm \\
0.002\end{array}$ & $0.683 \pm 0.048$ & $\begin{array}{c}0.986 \pm 0 \\
069\end{array}$ & $\begin{array}{c}1.019 \pm 0 \\
071\end{array}$ \\
\hline $30 \%$ & $\begin{array}{c}0.027 \pm 0 \\
001\end{array}$ & $\begin{array}{c}0.044 \pm \\
0.002\end{array}$ & $\begin{array}{c}0.067 \pm \\
0.002\end{array}$ & $\begin{array}{c}0.123 \pm \\
0.001\end{array}$ & $\begin{array}{c}0.184 \pm \\
0.001\end{array}$ & $\begin{array}{c}0.257 \pm \\
0.001\end{array}$ & $\begin{array}{c}0.350 \pm \\
0.001\end{array}$ & $\begin{array}{c}0.455 \pm \\
0.003\end{array}$ & $\begin{array}{c}0.537 \pm \\
0.001\end{array}$ & $0.218 \pm 0.035$ & $\begin{array}{c}0.315 \pm 0 \\
051\end{array}$ & $\begin{array}{c}3.261 \pm 0 \\
526\end{array}$ \\
\hline
\end{tabular}


Table.3 Proximate composition of Spirulina platensis species

\begin{tabular}{|c|c|}
\hline Parameter & Spirulina platensis \\
\hline \% Moisture & $88.83 \pm 0.41$ \\
\hline Dry Weight (g/L) & 0.98 \\
\hline Carbohydrate $(\mathbf{m g} \%)$ & $11.07 \pm 0.18$ \\
\hline Protein $(\mathrm{mg} \%)$ & $47.59 \pm 0.20$ \\
\hline Nitrogen Content & $7.62 \pm 0.03$ \\
\hline Chlorophyll a ( $\mu \mathrm{g} / \mathrm{L})$ & $2.4 \pm 0.007$ \\
\hline Chlorophyll b $(\mu \mathrm{g} / \mathrm{L})$ & $6.19 \pm 0.002$ \\
\hline Total Chlorophyll $(\mu \mathrm{g} / \mathrm{L})$ & $8.59 \pm 0.09$ \\
\hline
\end{tabular}

Table.4 Lipid content of Spirulina platensis microalgae culture

\begin{tabular}{|c|c|c|}
\hline Microalgae Species & Optimized Treatment & Lipid Content (\%) \\
\hline Spirulina platensis & BG-11 Media & $23.924 \pm 0.59$ \\
\hline & $30^{\circ} \mathrm{C}$ Temperature & $22.668 \pm 3.93$ \\
\hline $50 \%$ Light Intensity & $21.265 \pm 1.07$ \\
\hline
\end{tabular}

\section{Chlorophyll content}

In the present investigation chlorophyll-a pigment was in Spirulina platensis was found $2.4 \mu \mathrm{g} / \mathrm{L}$ similar trend was followed for the Chlorophyll-b and Total chlorophyll content. Chlorophyll-b content in Spirulina platensis was found $6.2 \mu \mathrm{g} / \mathrm{L}$. The total chlorophyll was found in Spirulina platensis was found 8.59 $\mu \mathrm{g} / \mathrm{L}$ indicated in Table 3. Similar result was found by investigated carried out by Hosikian, et al., (2012).

\section{Effect of optimized growth parameter on lipid/oil content}

Lipids serve as a storage material in living organisms and on oxidation process it produces more energy. Effect of optimized parameters for microalgae growth was studied on effect of lipid productivity. In this present study, for Spirulina platensis higher lipid content $(23.92 \%)$ was found in BG-11 growth medium with temperature $30^{\circ} \mathrm{C}(22.67$ $\%)$ and for light intensity $50 \%(21.26 \%)$ as indicated in Table 4. Spirulina platensis is fresh water microalgae and mainly enrich in protein and also with carbohydrates and lipid. From this study it is revealed that Spirulina platensis a fresh water microalgae grown in laboratory scale photobioreactor for growth parameter optimization. The result found that the optimized growth parameter for Spirulina plantnes was found in BG-11 medium with temperature $30{ }^{\circ} \mathrm{C}$ and light intensity $50 \%$.For biochemical characterization it was found that Spirulina Plantnes possesses higher value of protein content and had enormous value for pharmaceutical and nutraceutical. It can be used as best alternative food supplement for human.

\section{Acknowledgements}

Authors are thankful to Dr. B.A. Golakiya, Professor and Head, Department of Biotechnology, Junagadh Agricultural University, Junagadh for providing necessary lab facilities. The first author is grateful to the Department of Science and Technology for Inspire Fellowship, Government of India. New Delhi. 


\section{References}

Association of Official Analytical Chemists (AOAC). Official methods of analysis of the Association of Official Analytical Chemists. 14th ed. Arlington: 1984.

Bagchi, S. K., Rao, P. S. and Mallick, N. 2015. Bioreso. Technol. 180:207-213.

Colla,

L.M., C.O. Reinehr, C.J. Reichert, A.V. Costa. 2007. Bioreso. Technol., 98: 1489-1493.

Griffiths, M. J., Garcin, C., Van, R. P. and Harrison, S. 2011. J. of Microbiological Methods. 85:119-123.

Guillard, R. R. and Sieracki, M. S. 2005. Algal culturing techniques. Elsevier Academic Press: 239-252.

Hill, J., Nelson, E., Tilman, D., Polasky, S. and Tiffany, D. Proceeding of National Academy of Science. 103:11206-11210.

Hosikian, A., Lim, S., Halim, R., and Danquah, M. K. 2010. Int. j. of Chemical Engineering. 1:1-11.
Jeffrey, S. W. and Humphrey, G. F. 1975. Biochemistry and Physiology of Plants. 167:191-194.

Joshi, K.B., M.K. Mandavia and Golakiya, B.A. 2018. Int.J.Curr. Microbio. and Applied Sci. 7(04): 1439-1447. doi: https://doi.org/10.20546/ijcmas.

Mercz, T. 1994. PhD thesis, Murdoch University, Perth, Australia.

Pienkos, P. T. and Darzins, A. 2009. Biofuel Bioproducts Biorefinary.3:431-440.

Pulz, O. 2001.Applied Microbio. Biotechno. 57:287-293.

Ricketts, T. R. 1966. Phytochemistry, 5(1): 67-76.

Spolaore, P., Joannis-Cassan, C., Duran, E. and Isambert, A. 2006. J. of Biosci. and Bioengg. 101:87-96.

Wijffels, R. H., Barbosa, M. J. and Eppink, M. H. 2010. Biofuels, Bioproducts and Biorefining. 4:287-295.

\section{How to cite this article:}

Joshi, V.P., P.M. Chauhan, K.B. Joshi and Joshi, N.H. 2018. Growth Parameter Optimization and Biochemical Appraisals of Spirulina platensis. Int.J.Curr.Microbiol.App.Sci. 7(11): 160169. doi: https://doi.org/10.20546/ijcmas.2018.711.020 